\title{
Tobacco smoking and smoking cessation in times of COVID-19
}

\author{
Jan M. Elling', Rik Crutzen', Reinskje Talhout², Hein de Vries
}

\begin{abstract}
INTRODUCTION Tobacco smoking may increase susceptibility to and severity of coronavirus disease 2019 (COVID-19). This information may influence smoking cessation related beliefs in smokers.

METHODS Online questionnaires were answered from 26 March to 3 April 2020 in the Netherlands by 340 smokers willing to quit smoking. Beliefs regarding (quitting) smoking and (consequences of) the coronavirus are described and associations with motivation to quit due to the coronavirus are reported.

RESULTS While $67.7 \%$ of the smokers indicated that the coronavirus did not influence the number of cigarettes smoked per day, 18.5\% smoked less cigarettes and $13.8 \%$ smoked more cigarettes per day due to the coronavirus. One-third of the smokers were more motivated to quit smoking due to the coronavirus. Motivation to quit due to the coronavirus was positively associated with beliefs about the coronavirus as a serious threat, being at high risk of catching the coronavirus and developing severe illness, smokers being at higher risk than non-smokers, quitting smoking to reduce complaints, the social environment endorsing quitting, and perceived stress.

CONCLUSIONS Subgroups of smokers may be receptive to smoking cessation advice due to COVID-19. Because of the measures taken to reduce the spread of the virus (e.g. stay at home as much as possible), personalized digital health interventions may be particularly suitable to reach smokers at home.
\end{abstract}

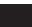

\section{AFFILIATION}

1 Department of Health Promotion, Maastricht University/CAPHRI, Maastricht, The Netherlands

2 Centre for Health Protection, National Institute for Public Health and the Environment (RIVM),

Bilthoven, The Netherlands

\section{CORRESPONDENCE TO}

Jan M. Elling. Department of Health Promotion, Maastricht University/ CAPHRI, P.O. Box 616, Maastricht, 6200 MD, The Netherlands. E-mail: m.elling@maastrichtuniversity.nl ORCID ID: https://orcid.org/00000002-1402-1860

\section{KEYWORDS}

smoking cessation, tobacco, smoking, coronavirus, COVID-19, SARS-CoV-2
\end{abstract}

\section{Received: 7 May 2020}

Revised: 22 May 2020

Accepted: 22 May 2020

\section{INTRODUCTION}

Coronavirus disease 2019 (COVID-19) is a respiratory infection caused by severe acute respiratory syndrome coronavirus 2 (SARS-CoV-2), also known as coronavirus. The COVID-19 pandemic has determined daily life in most countries in the past weeks/months and there is no end in sight.

The current situation may influence (beliefs about) smoking behavior. Smokers may smoke more tobacco because of higher stress levels due to the crisis situation ${ }^{1}$. Yet, smokers may also perceive increased susceptibility to and severity of COVID-19, potentially increasing motivation to quit.

The coronavirus may pose an additional threat to smokers. First, the hand-to-mouth movement while smoking can facilitate viral infection. Public health measures strongly advise to avoid touching eyes, nose and mouth, since hands can transfer the virus to the face and from there enter the body. Second, tobacco smoke damages the lungs of the smoker and compromises the immune system resulting in an increased risk for respiratory infections and negative disease progressions ${ }^{2}$.

There is distinct evidence that smoking status is associated with severity and mortality of COVID-19. A recent meta-analysis revealed that current smoking increases the risk of severe COVID-19 by around twofold (pooled OR=1.98; 95\% CI: 1.29-3.05) . Mehra et al. ${ }^{4}$ evaluated the relationship of smoking status and in-hospital mortality among 8910 
hospitalized COVID-19 patients. Current smoking status was found to be independently associated with an increased risk of death $(\mathrm{OR}=1.79$; $95 \%$ CI: 1.29 2.47).

Smokers may be influenced by (news) reports on these findings about the link between smoking and COVID-19. Therefore, this study describes Dutch smokers' perceptions regarding susceptibility to and severity of COVID-19 and its effect on smoking behavior (i.e. number of cigarettes smoked) and difficulty to quit smoking. Associations between beliefs and motivation to quit due to the (consequences of) coronavirus are reported. As there may be gender differences in psychological responses to crises, differences in beliefs between men and women are also described ${ }^{5}$.

\section{METHODS}

From 26 March to 3 April 2020, a questionnaire was distributed to smokers willing to quit smoking. Inclusion criteria were that participants were aged $\geq 18$ years, have smoked cigarettes in the past 7 days, and were motivated to quit smoking within 5 years. The sample was provided by Flycatcher, a Dutch ISO certified internet research agency with more than 10000 voluntary members. In return for full participation, panel members received a lottery ticket and points, which can be redeemed for gift vouchers. Informed consent was obtained online. The questionnaire was part of an overarching project on digital smoking cessation interventions for which ethical approval was granted by the institutional ethical review committee (FHML-REC/2019/072). Potential participants were selected for invitation based on the inclusion criteria. Flycatcher sent an invitation e-mail to 463 members of their panel, of which $\mathrm{N}=340$ completed the questionnaire (response rate: $73 \%$ ).

The I-Change model ${ }^{6}$, aimed at explaining motivational and behavioral change by integrating various social-cognitive theories, served as a theoretical framework for the beliefs about smoking and the coronavirus and covered risk perception, attitude, social norms, self-efficacy, and action planning (Figure 1). All items were measured on a 5-point Likert scale ranging from 1='Strongly disagree' to $5=$ ='Strongly agree'. Means and $99.99 \%$ confidence intervals for the beliefs were calculated and are reported as diamonds in the left-hand panel of Figure 1.

Correlations between the individual items and motivation to quit (MTQ) due to (consequences of) the coronavirus were calculated. MTQ due to the coronavirus (i.e. 'Because of the coronavirus, I'm now more motivated to quit smoking') was measured on a 5 -point Likert scale ranging from $1=$ 'Strongly disagree' to $5=$ 'Strongly agree'. The association strength is reported in the right-hand panel of Figure 1 . The diamonds visualize the correlation coefficients with $95 \%$ confidence intervals.

The confidence interval-based estimation of relevance (CIBER) approach was employed to visualize the results ${ }^{7}$. The tool is part of the "userfriendlyscience" ${ }^{8}$ package for the statistical computing environment $\mathrm{R}$.

Furthermore, changes in smoking behavior due to the coronavirus (i.e. 'Because of the coronavirus, I now smoke more/less a day') were measured with response options being $1=$ 'Less cigarettes', $2=$ 'Unchanged', and 3='More cigarettes'. For response options 1 and 3 , the exact number of cigarettes smoked less or more was measured as well.

Demographics were assessed by age, gender, and education level (low, intermediate, high). Cigarette dependence was assessed by the Fagerström Test ${ }^{9}$. The six items of the scale were summed into an overall score ranging from 0 to 10 . Willingness to quit smoking was assessed by asking participants when are they planning to quit smoking (within 1 month, within 6 months, within 1 year, within 5 years).

\section{RESULTS}

\section{Sample characteristics}

The mean age of participants was 49 years $(\mathrm{SD}=13$; range $=21-80)$ and the majority was female $(60.9 \%)$. Of the participants, $21.8 \%$ had a low level of completed education (i.e. no education or primary education), $42.6 \%$ an intermediate level (i.e. secondary education), and $35.6 \%$ a high level (i.e. tertiary education). The mean cigarette dependence was moderate $(\mathrm{M}=3.8 ; \mathrm{SD}=2.5)$. Participants smoked an average of $88.8(\mathrm{SD}=67.9)$ cigarettes in the past 7 days. Sixty-three participants $(18.5 \%)$ were willing to quit within 1 month, $124(36.5 \%)$ were willing to quit within 6 months, 91 (26.8\%) were willing to quit 
Figure 1. Means of beliefs and associations with motivation $\left(R^{2}=0.66-0.77\right)$ to quit smoking due to the coronavirus

The coronavirus is a serious threat to my heath *
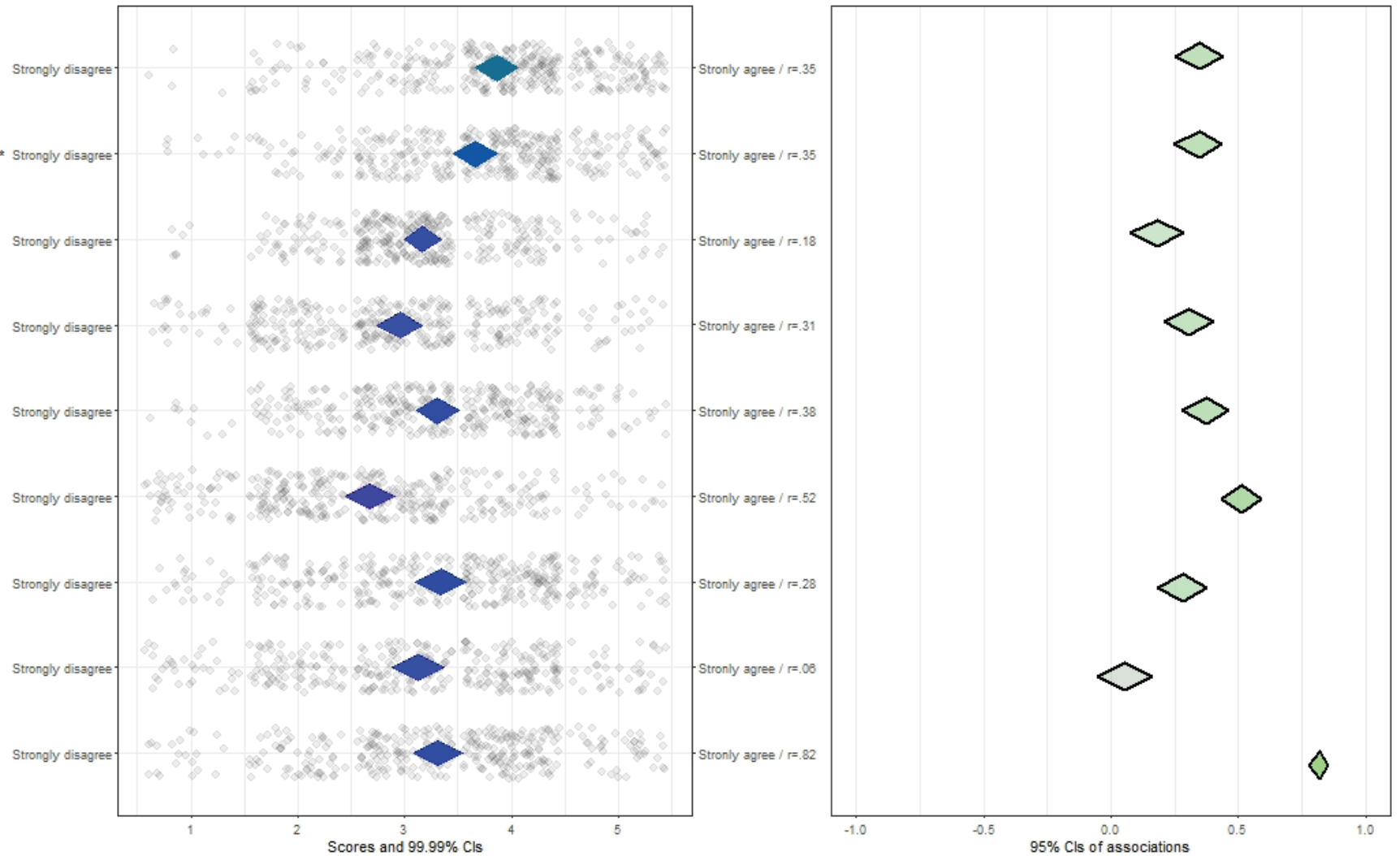
within 1 year and $62(18.2 \%)$ were willing to quit within 5 years.

\section{Smoking and the coronavirus}

Of the participants, $33.8 \%$ were more motivated to quit smoking because of the coronavirus and $66.2 \%$ were not more motivated to quit due to the coronavirus. Of our sample, $18.5 \%$ indicated that they smoke less cigarettes due to the coronavirus $(\mathrm{M}=4.1$ cigarettes/day, $\mathrm{SD}=3.3), 13.8 \%$ indicated that they smoke more cigarettes $(\mathrm{M}=7.3$ cigarettes/ day, $\mathrm{SD}=8.2$ ), and $67.7 \%$ did not alter the number of cigarettes.

The CIBER analyses (Figure 1) reveal that MTQ due to the coronavirus was positively associated with beliefs about the coronavirus as a serious threat, being at high risk of catching the coronavirus and developing severe illness, smokers being at higher risk than non-smokers, quitting smoking to reduce complaints, the social environment endorsing quitting, and perceived stress. All beliefs reported in Figure 1, except for self-efficacy ('Because of the coronavirus, I find it hard to quit smoking'), were significantly $(\mathrm{p}<0.01)$ positively associated with MTQ due to the coronavirus. These items together explained $66-77 \%$ of the variance in MTQ due to coronavirus.

Analysis of variance also confirmed that smokers motivated to quit were significantly $(\mathrm{p}<0.01)$ more convinced of all these items than smokers not motivated to quit.

\section{Differences in beliefs about smoking and the coronavirus between men and women}

Men and women differed statistically significantly on two items. Women compared to men indicated that they experience more stress in their daily life because of the coronavirus $[\mathrm{F}(1,338)=11.94 ; \mathrm{p}<0.001$; $\eta_{\mathrm{p}}^{2}=0.034 ; 95 \%$ CI: $\left.0.006-0.080\right]$ and that they found it more difficult to quit smoking because of the coronavirus $\left[\mathrm{F}(1,338)=11.47 ; \mathrm{p}<0.001 ; \eta_{\mathrm{p}}^{2}=0.033\right.$; 95\% CI: 0.006-0.078].

\section{DISCUSSION}

One-third of our smokers were more motivated to quit smoking due to the coronavirus. Our results reveal which beliefs may be especially important to incorporate in smoking cessation interventions in times of COVID-19, such as the risks of being a smoker, and support from their social environment.

Since people are advised to stay at home in many countries to reduce the spread of the coronavirus, digital health smoking cessation interventions form an effective way to reach smokers ${ }^{10}$. In order to reach as many smokers as possible and reduce the spread of the coronavirus, we advocate evidencebased (digital health) interventions that are easily accessible for all citizens and include the latest evidence about the relation between smoking and COVID-19.

\section{Limitations}

A limitation of this study is that we included all smokers willing to quit smoking within 5 years. Thus, the sample consists of smokers highly motivated to quit and smokers less motivated to quit. Inclusion criteria of only smokers who are contemplating or preparing to quit may lead to different results.

\section{CONCLUSIONS}

The results of the present study provide evidence that perceiving the coronavirus as a serious threat and acknowledging that smokers are at higher risk than non-smokers is associated with motivation to quit. Believing that quitting decreases that risk and that friends and family endorse quitting is also associated with motivation to quit.

\section{REFERENCES}

1. Kassel JD, Stroud LR, Paronis CA. Smoking, stress, and negative affect: correlation, causation, and context across stages of smoking. Psychol Bull. 2003;129(2):270-304. doi:10.1037/0033-2909.129.2.270

2. Arcavi L, Benowitz NL. Cigarette smoking and infection. Arch Intern Med. 2004;164(20):2206-2216. doi:10.1001/archinte.164.20.2206

3. Zhao Q, Meng M, Kumar R, et al. The impact of COPD and smoking history on the severity of Covid-19: A systemic review and meta-analysis. J Med Virol. 2020;17. doi:10.1002/jmv.25889

4. Mehra MR, Desai SS, Kuy S, Henry TD, Patel AN. Cardiovascular disease, drug therapy, and mortality in Covid-19. N Engl J Med. 2020. doi:10.1056/NEJMoa2007621

5. Lachlan KA, Spence PR, Nelson LD. Gender Differences in Negative Psychological Responses to Crisis News: The Case of the I-35W Collapse. Commun Res Rep. 2010;27(1):38-48. doi:10.1080/08824090903293601

6. De Vries H. An integrated approach for understanding health behavior; the I-change model as an example. 
Psychol Behav Sci Int J. 2017;2(2):555-585. doi:10.19080/pbsij.2.2

7. Crutzen R, Peters G-JY, Noijen J. Using Confidence Interval-Based Estimation of Relevance to Select Social-Cognitive Determinants for Behavior Change Interventions. Front Public Health. 2017;5(165). doi:10.3389/fpubh.2017.00165

8. Peters G-JY. userfriendlyscience: Quantitative analysis made accessible. https://cran.r-project.org/web/ packages/userfriendlyscience/userfriendlyscience.pdf. Published 25 September 2018. Accessed May 9, 2020.

9. Heatherton TF, Kozlowski LT, Frecker RC, Fagerstrom KO. The Fagerstrom Test for Nicotine Dependence: a revision of the Fagerstrom Tolerance Questionnaire. British journal of addiction. 1991;86(9):1119-1127. doi:10.1111/j.1360-0443.1991.tb01879.x

10. Cheung KL, Wijnen B, de Vries H. A review of the theoretical basis, effects, and cost effectiveness of online smoking cessation interventions in the Netherlands: A mixed-methods approach. J Med Internet Res. 2017;19(6):e230. doi:10.2196/jmir.7209

CONFLICTS OF INTEREST

The authors have completed and submitted the ICMJE Form for Disclosure of Potential Conflicts of Interest and none was reported.

FUNDING

There was no source of funding for this research.

PROVENANCE AND PEER REVIEW

Not commissioned; externally peer reviewed. 